\title{
In-vivo and In-vitro Anti-Acinetobacter Baumannii Activity of Citrate-Capped Silver Nanoparticles
}

\author{
Ibtesam Ghadban Auda $^{1 \llbracket}$, Istabreq Mohamed Ali Salman ${ }^{1 \llbracket}$, Dalal Abed Al-Sattar ${ }^{1 \llbracket}$, \\ Jameelah Ghadban Oduha ${ }^{2}$ \\ ${ }^{1}$ Department of Biology, College of Science, Mustansiriyah University, Iraq. \\ ${ }^{2}$ Department of Microbiology, Al-Kindy College of Medical - Baghdad University, Iraq. \\ C Corresponding authors. E-mail: stbg@uomustansiriyah.edu.iq
}

Received: Nov. 20, 2020; Accepted: May 24, 202I; Published: Aug. 16, 2021

Citation: Ibtesam Ghadban Auda, Istabreq Mohamed Ali Salman, Dalal Abed Al-Sattar, and Jameelah Ghadban Oduha, In-vivo and In-vitro AntiAcinetobacter Baumannii Activity of Citrate-Capped Silver Nanoparticles. Nano Biomed. Eng., 202I, I3(3): 229-239.

DOI: 10.5101/nbe.v13i3.p229-239.

\begin{abstract}
Silver nanoparticles (AgNPs) are of potential interest because of their effective antibacterial and antiviral activities. Capping agents are used for exhibiting a better antibacterial activity than uncapped Ag NPs. There are very few reports that have shown the usage of AgNPs for in-vivo antibacterial therapy. Citrate-capped silver nanoparticles were synthesized chemically by citrate reduction method; the size of Cit-AgNPs was determined by an atomic force microscope (AFM) and was between 15 - $90 \mathrm{~nm}$. Acinetobacter baumannii (A. baumannii) isolates were the only sensitive species to CitAgNPs. MICs and MBC of Cit-AgNPs were determined by using A. baumannii. The results showed an additive effect of Cit-AgNPs. Four mice groups were infected with a sub-lethal dose of A. baumannii intraperitoneally, IP. The single daily dose of Cit-AgNPs and imipenem plus Cit-AgNPs combination were administered IP. Imipenem and phosphate buffer saline (PBS) was used as positive control and negative control, respectively. Interestingly, only the PBS-treated group showed growth of A. baumannii in the liver and spleen of sacrificed mice. Histopathologically, Cit-AgNPs showed antibacterial activity and had an additive effect when combined with imipenem in vivo and in vitro. Moreover, the Cit-AgNPs showed dose-dependent activity and the organs differed in the illumination of the toxicity effect of Cit-AgNPs even after high dose administration. In conclusion, Cit-capped AgNPs had antibacterial activity against multiple drug resistant (MDR) A. baumannii but not against K. pneumoniae and E. coli. Cit-capped AgNPs increased the inhibition zone of imipenem in additive effect; the minimum inhibitory concentration and the minimum bactericidal concentration of Citcapped AgNPs were relatively low. Cit-capped AgNPs eliminated A. baumannii infection in vivo when it was given alone or in combination with imipenem. The cytotoxicity of Cit-AgNPs was dosedependent and the organs differed in the illumination of the inflammatory effect of Cit-AgNPs after high dose administration. It is not recommended to use Cit-capped AgNPs systemically despite their valuable additive antibacterial effect especially with a high dose and the combination with imipenem, Topical administration needs to be evaluated.
\end{abstract}

Keywords: Citrate capped silver nanoparticles, Acinetobacter baumannii, RecA gene, In vivo, In vitro

\section{Introduction}

The appearance of multidrug-resistant (MDR) bacteria is an international problem for human health. Biological, chemical, and physical properties of nanoparticles making them probable alternatives to some antimicrobial agents [1, 2]. Silver nanoparticles (AgNPs) have potent antiviral and antibacterial activities [3]. Starting from the 18th century, silver in the form of silver sulfadiazine, silver nitrate, etc., has 
been used for the management of wounds, burns, and maybe other infections. Thereby this feature led to the incorporation of silver into a variety of germicidal preparations [4-6].

Silver nanoparticles have been synthesized by a variety of methods, such as silver ions reduction on functionalized silica surface $[7,8]$, reduction and capping of silver ions by citrate [3], and reduction of silver ions by nitrate reductase enzyme [9]. The antibacterial activities of the AgNPs varied according to the different synthesis methods. On the other hand, capping agents are nanoparticles stabilizing agent, and exhibiting antimicrobial activity better than uncapped AgNPs [10, 11].

Regarding the action mechanism, a variety of hypotheses have been suggested by many authors. One study mechanism suggests that silver nanoparticles affect the permeability of the bacterial cell wall due to the deposition of silver [12] and electron-dense granules creation [13]. Other studies have shown DNA failure to replicate prior silver ions treatment and enzymatic inactivation by reaction with thiol groups [14]. Extensively proton leakage of membranes occurred in E. coli and Vibrio cholerae leading to the collapse of the proton motive force $[15,16]$.

The synthesis of metal colloid required a reductant, the commonly used one is citrate ion. Silver nanocrystallites interact strongly with the surface of citrate ions. Comparing with radiological and chemical synthesis methods. The slow growth of crystals resulting from citrate ions and silver surface interaction is distinctive. Citrate ions addition to silver nitrate results in the complex formation of citrate with silver ions to produce citrate-capped silver nanoparticles, CitAgNPs [17]. Although the silver ions' antimicrobial activity is well-known, few works report the utilization of the AgNPs as an antibacterial agent for in vivo therapy [3]. The study aims to evaluate the antibacterial activities of Cit-AgNPs in vivo and in vitro as well as to evaluate the effect of Cit-AgNPs histopathologically on some vital organs.

\section{Experimental \\ Bacterial isolates}

Four A. baumannii isolates identified by the Vitek system were isolated from wounds of patients admitted to Baghdad hospitals $\backslash$ Iraq. Four of uropathogenic K. pneumoniae and four of uropathogenic E. coli isolates were obtained from the same mentioned hospital and identified by the Vitek system.

\section{Molecular detection of $\mathrm{A}$. baumannii isolates}

The recA gene (a housekeeping gene) was used for molecular detection of Acinetobacter baumannii using specific primers [18] forward primer, 5-CCTGAATCTTCGGTAAAAC-3, and reverse primer, 5-GTTTCTGGGCTGCCAAACATTAC-3. Template DNA was prepared by the boiling method by Auda et al. [19]. Briefly, loopful bacterial culture was suspended in $200 \mu \mathrm{l}$ of lysis buffer containing $1 \%$ Triton X-100, 0.5\% Tween 20, $10 \mathrm{mM}$ Tris- $\mathrm{HCl}(\mathrm{pH}=$ 8 ), and $1 \mathrm{mM}$ EDTA and incubated for 3 minutes in a boiling water bath. After centrifugation for 2 minutes at $10,000 \times \mathrm{g}$ to sediment the debris. After centrifugation, the supernatant was used as template DNA. A negative control is the PCR mixture without the template DNA while the positive control is the PCR mixture with A. baumannii ATCC DNA as atemplate (Baghdad University-College of Science).

\section{Antimicrobial susceptibility testing of $A$. baumannii}

It was performed by the disk diffusion method according to the CLSI guidelines [20]. Five families of the antimicrobial were used. The following discs $(\mu \mathrm{g} / \mathrm{disc})$, were used CP: ciprofloxacin $(5 \mu \mathrm{g}), \mathrm{CT}$ : cefotaxime $(30 \mu \mathrm{g})$, IP: imipenem $(10 \mu \mathrm{g})$, PI: pipracillin+ tazobactam $(100 / 10 \mu \mathrm{g})$, TZ: ceftazidime $(30 \mu \mathrm{g})$, gentamicin $(10 \mu \mathrm{g})$, amoxicillin + clavulanic acid $(20 / 10 \mu \mathrm{g})$. These discs were obtained from Ninawa drug industries (NDI-Iraq).

\section{Citrate-capped silver nanoparticles synthesis}

Citrate-capped AgNPs (Cit-AgNPs) were prepared according to a method of Atta et al. [21] with little modifications. Briefly, $0.018 \%$ of the $\mathrm{AgNO}_{3}$ solution in deionized water was boiled, and $1 \%$ of sodium citrate solution was added dropwise under vigorous stirring. The solution was kept at boiling temperature for 10 minutes then kept in a boiling water bath for about 30 minutes. The change of color to a grayishyellow indicated the formation of AgNPs and then the solution was cooled to room temperature before been used for further experiments. The prepared CitAgNPs were tested for stability at room temperature and refrigerator for no more than one week to ensure its stability at these two conditions. The prepared CitAgNPs was stable until 5 days after preparation. 


\section{Determination of Cit-AgNPs size by atomic force microscope (AFM)}

Synthesized Cit-AgNPs were precipitated on the slide, dried then sent to the Department of Chemistry/ University of Baghdad to determine the Cit-AgNPs size by atomic force microscope [22].

\section{Antimicrobial activity of Cit-AgNPs}

The antimicrobial activity of synthesized Cit-AgNPs was tested using 3 groups of pathogenic bacteria, A. baumannii, K. pneumoniae, and E. coli (four isolates of each group). The method by which the isolates were tested was the agar well diffusion method [3]. The effect of imipenem $(5 \mu \mathrm{g} / \mathrm{ml})$ and Cit-AgNPs (11.25 $\mu \mathrm{g} / \mathrm{ml}$ ) combination on A. baumannii was also tested. The obtained inhibition zones resulted from such combination were compared with that resulted from each separately.

\section{Determinations of MICs and MBCs}

MICs of Cit-AgNPs were determined by using the broth microdilution method [23] with A. baumannii and two-fold dilutions of Cit-AgNPs (90, 45, 22.5, $11.25,5.625,2.81,1.4$, and $0.70 \mu \mathrm{g} / \mathrm{ml})$. The lowest concentration showing no visible growth was considered as the MIC. From the clear wells, $100 \mu \mathrm{l}$ of each sample was sub-cultured onto nutrient agar to determine the minimum bactericidal concentration (MBC).

\section{In-vivo experiment}

BALB/c mice were obtained from Pharmaceutical Censorship Center-Baghdad and housed at the animal house at Biology department/college of Science/ Mustansiriyah University. The used 25, 6-8 weeks' mice were divided randomly into 5 groups ( 5 mice each). All procedures with animals were performed in agreement with the animal handling institution's ethics. Four mice groups were infected with a sublethal dose of A. baumannii intraperitoneal, IP, $(1 \times$ $10^{7} \mathrm{CFU} /$ mouse). Single daily dose of Cit-AgNPs (3.5 $\mathrm{mg} / \mathrm{kg}$ ) and imipenem plus Cit-AgNPs combination (5 $\mathrm{mg}+1.75 \mathrm{mg} / \mathrm{kg}$ ) were administered IP. Imipenem (10 $\mathrm{mg} / \mathrm{kg}$ ) and phosphate buffer saline (PBS) was used as the positive control and negative control respectively. The 4 groups received this treatment daily for 3 days as well as a $5^{\text {th }}$ group receive only Cit-AgNPs (3.5 mg/ $\mathrm{kg}$ ) once daily for 3 days. An additional group ( $6^{\text {th }}$ group) serves as normal healthy control. The first 4 mice groups were sacrificed on day 4 , and the liver and spleen were aseptically separated and homogenized in sterile PBS. The homogenate was plated in on nutrients to determine the bacterial load in the liver and spleen. The $5^{\text {th }}$ group was left for 15 days then were sacrificed. Vital organs including the liver, spleen, and kidney were separately collected and the histopathological section was made and stained with hematoxylin and eosin and examined by a microscope [3]. Fig. 1 shows the in-vivo experimental procedure.

\section{Result and Discussion \\ Molecular identification of $A$. baumannii isolates}

A. baumannii isolates were identified by recA gene amplification with specific primers, the obtained amplicon was 425 bp (Fig. 2).

\section{Antimicrobial susceptibility testing}

The antimicrobial susceptibility test of A. baumannii isolates revealed that four tested isolates were resistant to all antimicrobial tested except imipenem. On the bases of these results, A. baumannii was used as a model for in vivo experiments, and imipenem was used as a positive control. Genetically resourceful A. baumannii has grown to be endemic in hospitals, hence the genetic machinery of A. baumannii allows it to the rapid development of resistance. Therefore, Multidrug-resistant A. baumannii outbreaks in hospitals as a nosocomial infection are prevalent and were reported globally [24]. However, due to the recent unproductive therapeutic outcomes, the need to develop new antibacterial agents and understanding the role of other potential alternatives is necessary.

\section{Synthesis of citrate-capped silver nanoparticles}

Citrate-capped AgNPs (Cit-AgNPs) were prepared according to the chemical-boiling method as described above. The change of color to a grayish-yellow indicated the formation of Cit-AgNPs as shown in Fig. 3.

\section{Determination of Cit-AgNPs size by atomic force microscope}

An atomic force microscope is a type of microscope that provides pictures of atoms in or on surfaces. Like the scanning electron microscope (SEM), the purpose of the AFM is to stare at substances on the atomic level. It is commonly used in nanotechnology [22]. The obtained results of AFM revealed that the average diameter of Cit-AgNPs was $59.39 \mathrm{~nm}$ and the range of 


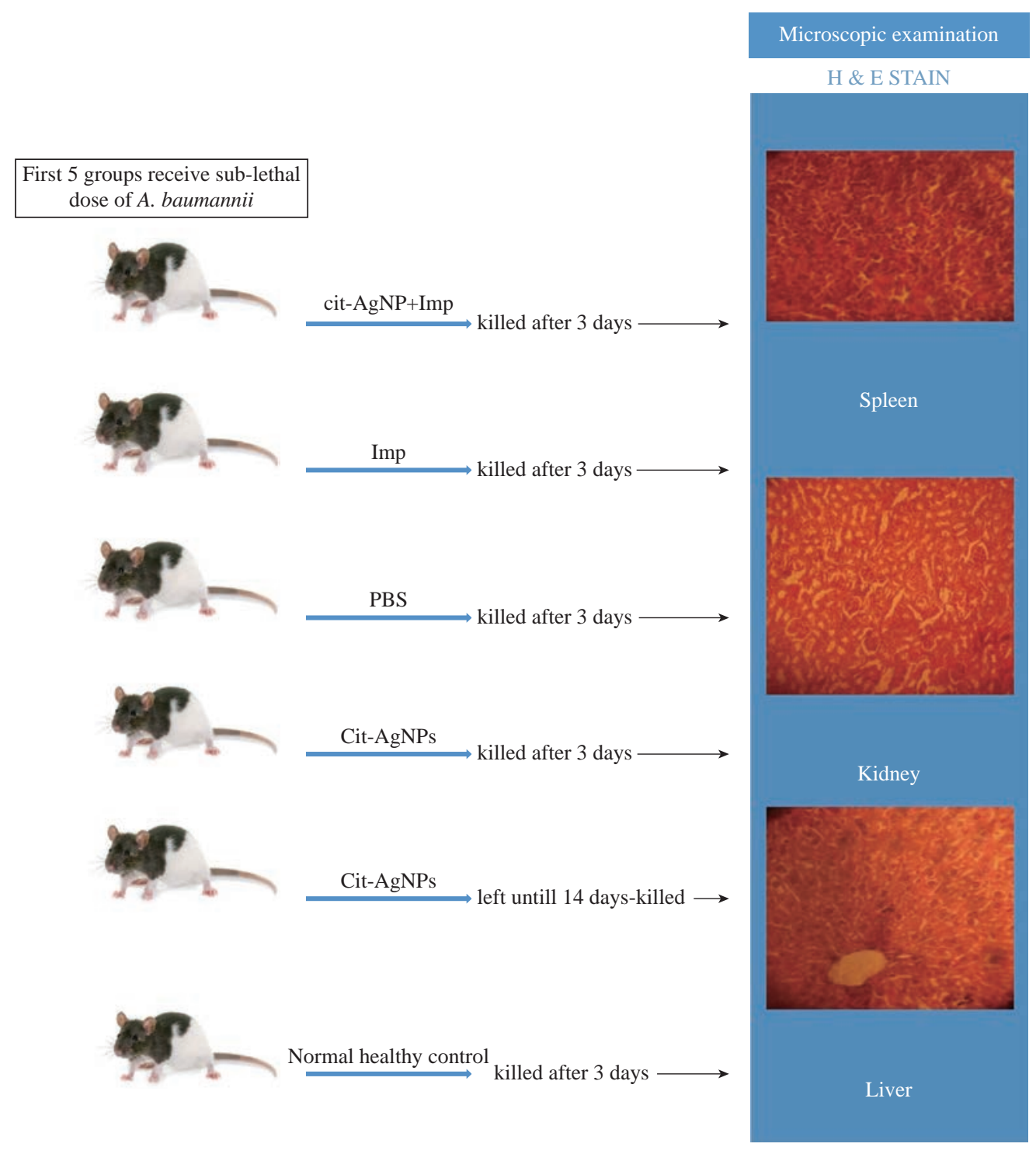

Fig. 1 Experimental procedure.

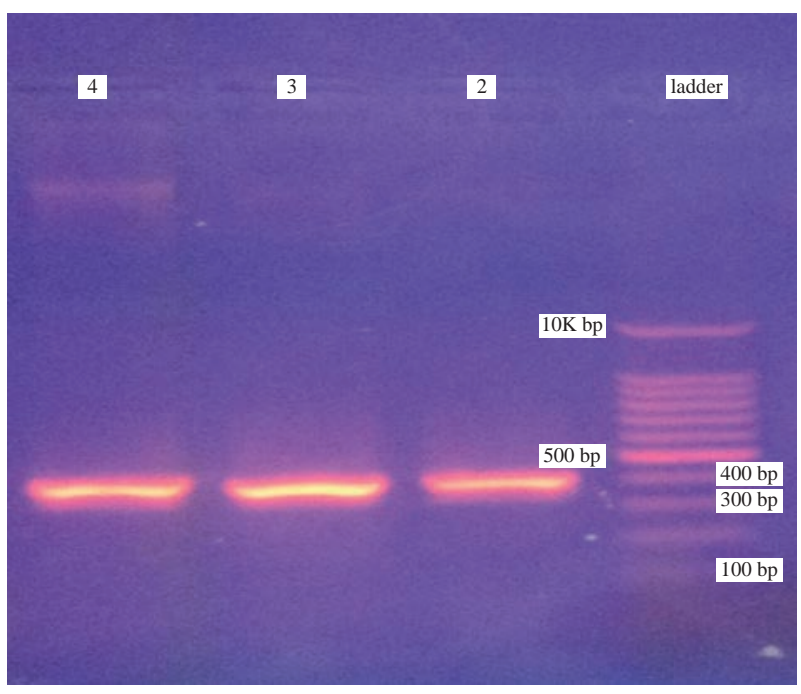

Fig. 2 Agarose gel electrophoretogram of recA gene PCR products: Lane 1 is a 100 bp molecular ladder; Lane 2, 3 and 4 are PCR products. All three lanes show bands of recA (425 bp). Agarose gel electrophoresis was performed using 1.5\% agarose gel and the run last for $90 \mathrm{~min}$ at $7 \mathrm{~V} / \mathrm{cm}$.
Cit-AgNPs size was between $15-90 \mathrm{~nm}$ while $50 \%$ of Cit-AgNPs size was $60 \mathrm{~nm}$. In a previous study, CitAgNPs size was between 10-55 nm [25], lower than that of this study, but the obtained Cit-AgNPs size was still at the nano-scale level. Table 1 and Figs. 3 and 4 summarized the Cit-AgNPs size as measured by AFM.

\section{Anti-A. baumannii activity of Cit-AgNPs}

Acinetobacter baumannii was the only sensitive group while K. pneumoniae and E. coli groups were resistant to Cit-AgNPs and showed no inhibition zone at all (Fig. 5 and Table 2).

The effect of imipenem and Cit-AgNPs combination on A. baumannii in vitro was tested. Interestingly, the Cit-AgNPs increased the inhibition zone of imipenem about $4 \mathrm{~mm}$ in 4 tested A. baumannii isolates (Fig. 6 and Table 2). It was reported that AgNPs have antibacterial activities at very low 

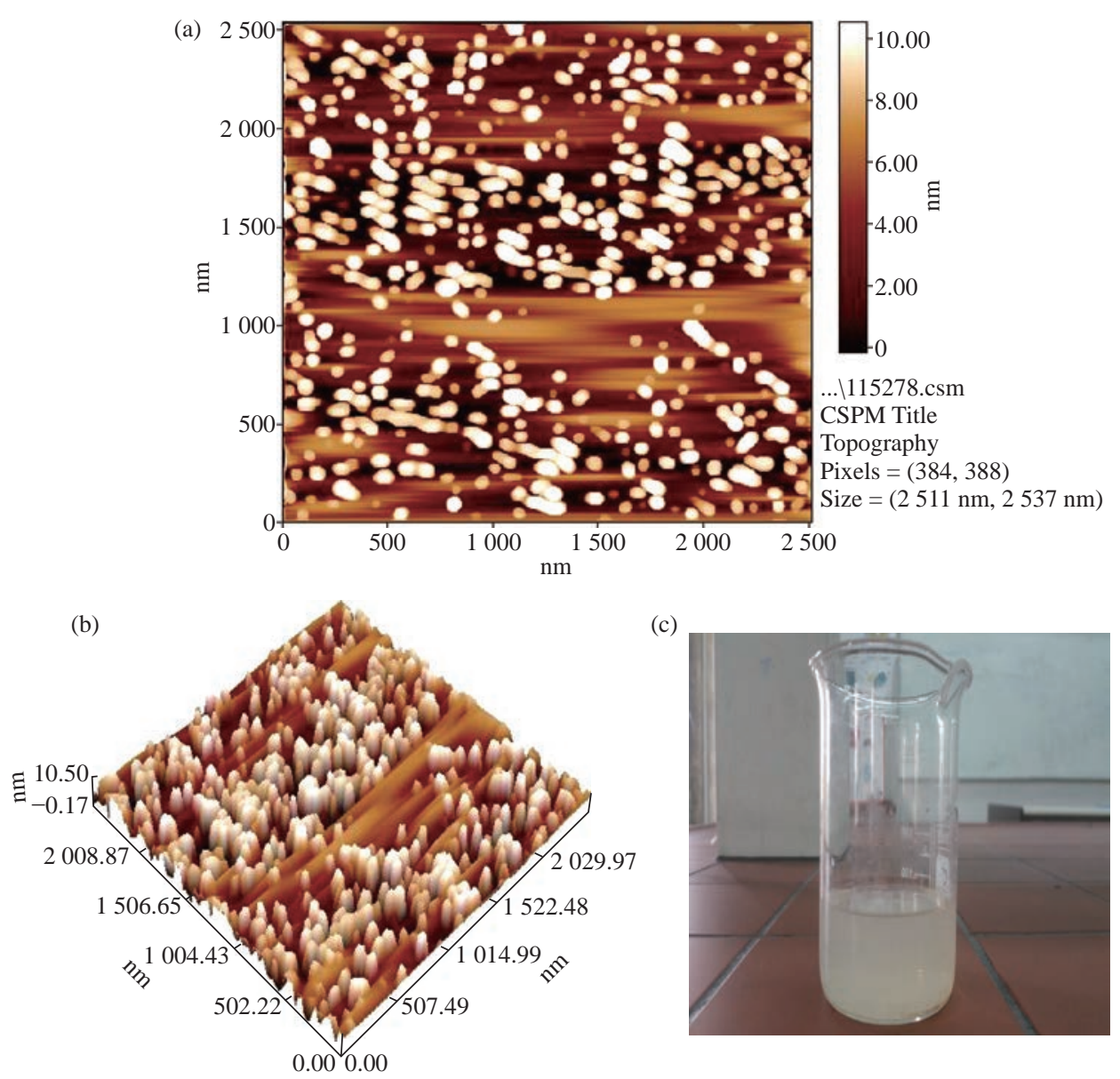

Fig. 3 Citrate-capped silver nanoparticles. (a) and (b) Citrate-capped silver nanoparticles size on the slide as captured by an atomic force microscope; (c) Prepared citrate-capped silver nanoparticles.

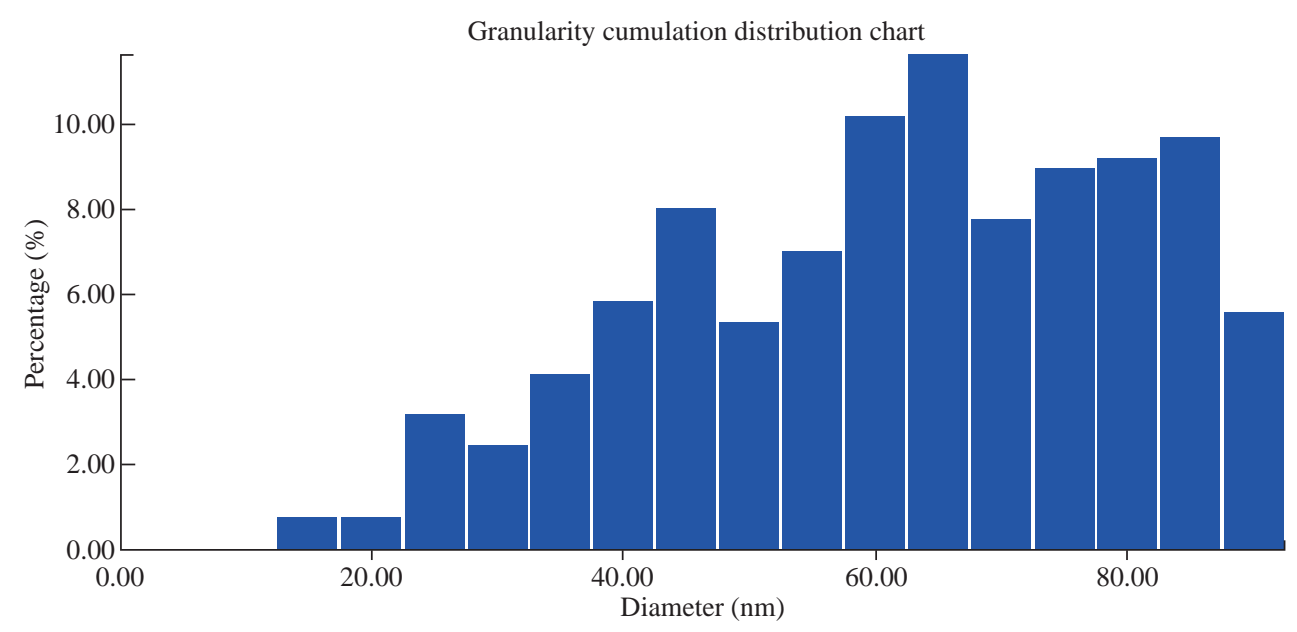

Fig. 4 Granularity cumulation distribution chart: Percentage of Cit-AgNPs according to the size.

concentrations and exhibit a synergistic effect with many other antibacterial drugs, such as penicillin, amoxicillin, erythromycin, and clindamycin [26]. Such an effect was observed when Cit-AgNPs combined with imipenem in this study and it was not reported previously.

\section{Determinations of MICs and MBCs of Cit- AgNPs against $A$. baumannii}

Four isolates of A. baumannii showed the same MIC value, $11.25 \mu \mathrm{g} / \mathrm{mL}$. while all except one isolate showed the same MBC value, $22.5 \mu \mathrm{g} / \mathrm{mL}$ (Table 3). Some researchers found that the MIC of AgNPs was 
Table 1 Distribution of prepared citrate-capped silver nanoparticles according to particle size

\begin{tabular}{cccccccccc}
\hline $\begin{array}{c}\text { Diameter (nm) } \\
<\end{array}$ & $\begin{array}{c}\text { Volume } \\
(\%)\end{array}$ & $\begin{array}{c}\text { Cumulation } \\
(\%)\end{array}$ & $\begin{array}{c}\text { Diameter (nm) } \\
<\end{array}$ & $\begin{array}{c}\text { Volume }(\%) \\
(\%)\end{array}$ & $\begin{array}{c}\text { Cumulation } \\
(\%)\end{array}$ & $\begin{array}{c}\text { Diameter (nm) } \\
<\end{array}$ & $\begin{array}{c}\text { Volume } \\
(\%)\end{array}$ & $\begin{array}{c}\text { Cumulation } \\
(\%)\end{array}$ \\
\hline 15.00 & 0.72 & 0.72 & 45.00 & 7.97 & 24.88 & 75.00 & 8.94 \\
20.00 & 0.72 & 1.45 & 50.00 & 5.31 & 30.19 & 75.60 \\
25.00 & 3.14 & 4.59 & 55.00 & 7.00 & 37.20 & 80.00 & 9.18 & 84.78 \\
30.00 & 2.42 & 7.00 & 60.00 & 10.14 & 47.34 & 85.00 & 9.66 \\
35.00 & 4.11 & 11.11 & 65.00 & 11.59 & 58.94 & 90.00 & 5.56 \\
40.00 & 5.80 & 16.91 & 70.00 & 7.73 & 66.67 & & 100.00 \\
\hline
\end{tabular}

Avg. diameter: $59.39 \mathrm{~nm} ; \leq 10 \%$ diameter: $30.00 \mathrm{~nm} ; \leq 50 \%$ diameter: $60.00 \mathrm{~nm} ; \leq 90 \%$ diameter: $80.00 \mathrm{~nm}$.

Table 2 Mean and SD of inhibition zone diameter of imipenem, Cit-AgNPs, and imipenem-Cit-AgNPs combination against A. baumannii

\begin{tabular}{cccc}
\hline Isolate No. & Imipenem mean + SD (mm) & Cit-AgNPs mean + SD (mm) & Imipenem + Cit-mean + SD (mm) \\
\hline 1 & $24+0.32$ & $13+0.39$ & $28+0.73$ \\
2 & $24+0.53$ & $16+0.50$ & $28+0.75$ \\
3 & $30+0.41$ & $15+0.50$ & $34+0.72$ \\
4 & $27+0.36$ & $15+0.38$ & $31+0.70$ \\
\hline
\end{tabular}

Cit-AgNPs: Citrate-capped silver nanoparticles
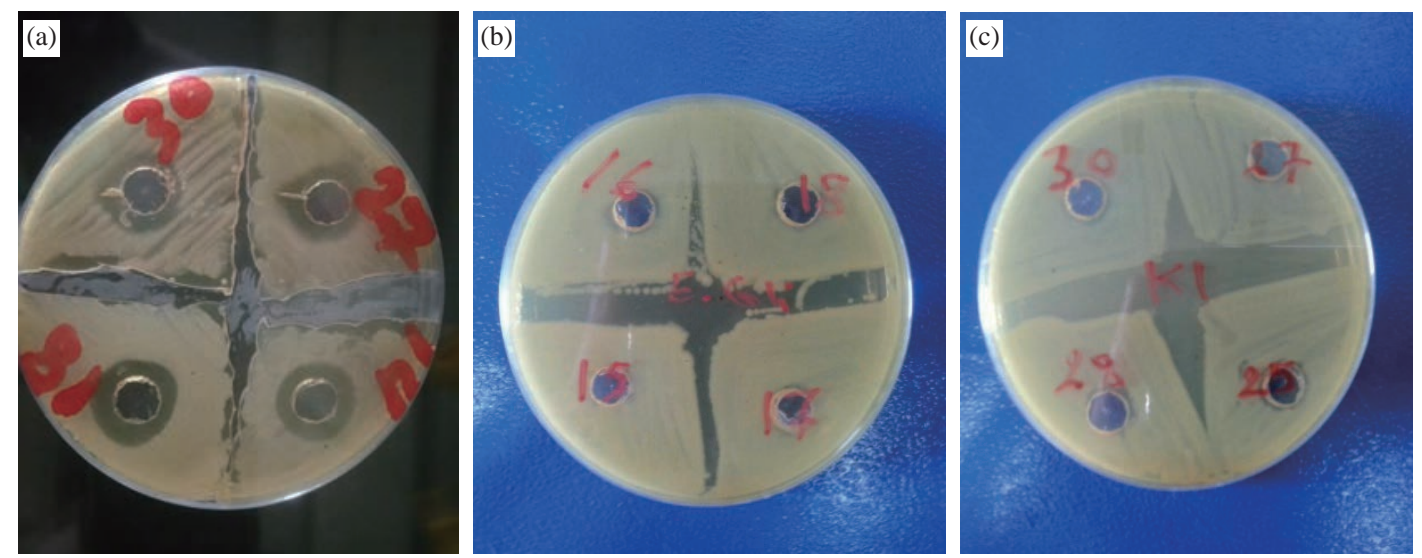

Fig. 5 Antibacterial effect of Cit-AgNPs on pathogenic bacteria: (a) A. baumannii (4 isolates), (b) E. coli (4 isolates), and (c) K. pneumoniae (4 isolates). Cit-AgNPs: Citrate-capped silver nanoparticles. There are inhibition zones around Cit-AgNPs wells only in the culture plate of $\mathrm{A}$. baumannii.
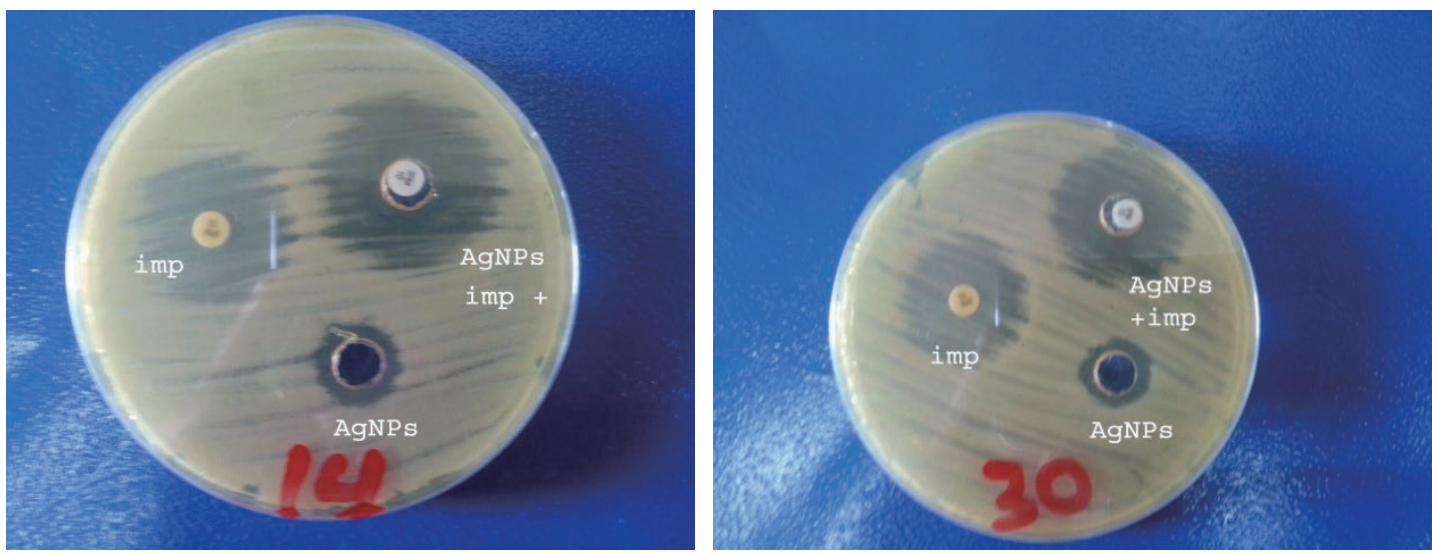

Fig. 6 Effect of imipenem - Cit-AgNPs combination against A. baumannii. Imp: Imipenem; AgNPs: Silver nanoparticles; AgNPs + imp: Silver nanoparticles + imipenem.

Table 3 The effect of citrate-capped silver nanoparticle concentrations on A. baumannii

\begin{tabular}{ccc}
\hline Isolate No. & MIC $(\mu \mathrm{g} / \mathrm{mL})$ & $\mathrm{MBC}(\mu \mathrm{g} / \mathrm{mL})$ \\
\hline 1 & 11.25 & 11.25 \\
2 & 11.25 & 22.5 \\
3 & 11.25 & 22.5 \\
4 & 11.25 & 22.5 \\
\hline
\end{tabular}

MIC: Minimum inhibitory concentration, MBC: minimum bactericidal concentration very low [26] and differ from that obtained by others who found it higher [3]. This diversity in results may be attributed to experiment design and the dilutions of AgNPs preparations that differ from each other according to experiment design.

\section{In-vivo experiment}

On the fourth day, the animals in 5 groups and 
the liver and spleen were plated on nutrient agar. The results revealed that only PBS-treated group showed growth of A. baumannii. In vivo, serum albumin interacts with uncapped AgNPs affecting the antibacterial activity of AgNPs. However, capped AgNPs [with citrate or poly(vinylpyrrolidone)] exhibited antibacterial activity in vivo due to minimized interactions with proteins of serum (3). Based on these facts, Cit-AgNPs was prepared to be used in vivo.

Histopathological study: the main targets of AgNPs are kidneys, spleen, and liver [27, 28]. The tissue of three vital organs of 5 groups was stained and examined microscopically. Kidneys of the imipenem treated group showed necrosis, degeneration of glomeruli, distal, proximal tubules cells and mucin secretion. Imipenem plus Cit-AgNPs group showed the same picture as the imipenem group. The pathological changes in these two groups may be due to A. baumannii infection and CitAgNPs at $1.75 \mathrm{mg} / \mathrm{kg}$ of this study have no detectable effect of AgNPs at low concentration with minimal side effect on kidneys that's belongs to large size of AgNPs [29]. The kidneys of mice groups treated with Cit-AgNPs and PBS reveal severe degeneration and necrosis, excessive mucin secretion in distill and proximal tubules, and loss of glomeruli structure and atrophy. At a high dose of $3.5 \mathrm{mg} / \mathrm{kg}$ Cit-AgNPs, as considered by some researchers [30], there are marked histopathological lesions that were observed in kidneys of the Cit-AgNPs group of this study and as reported
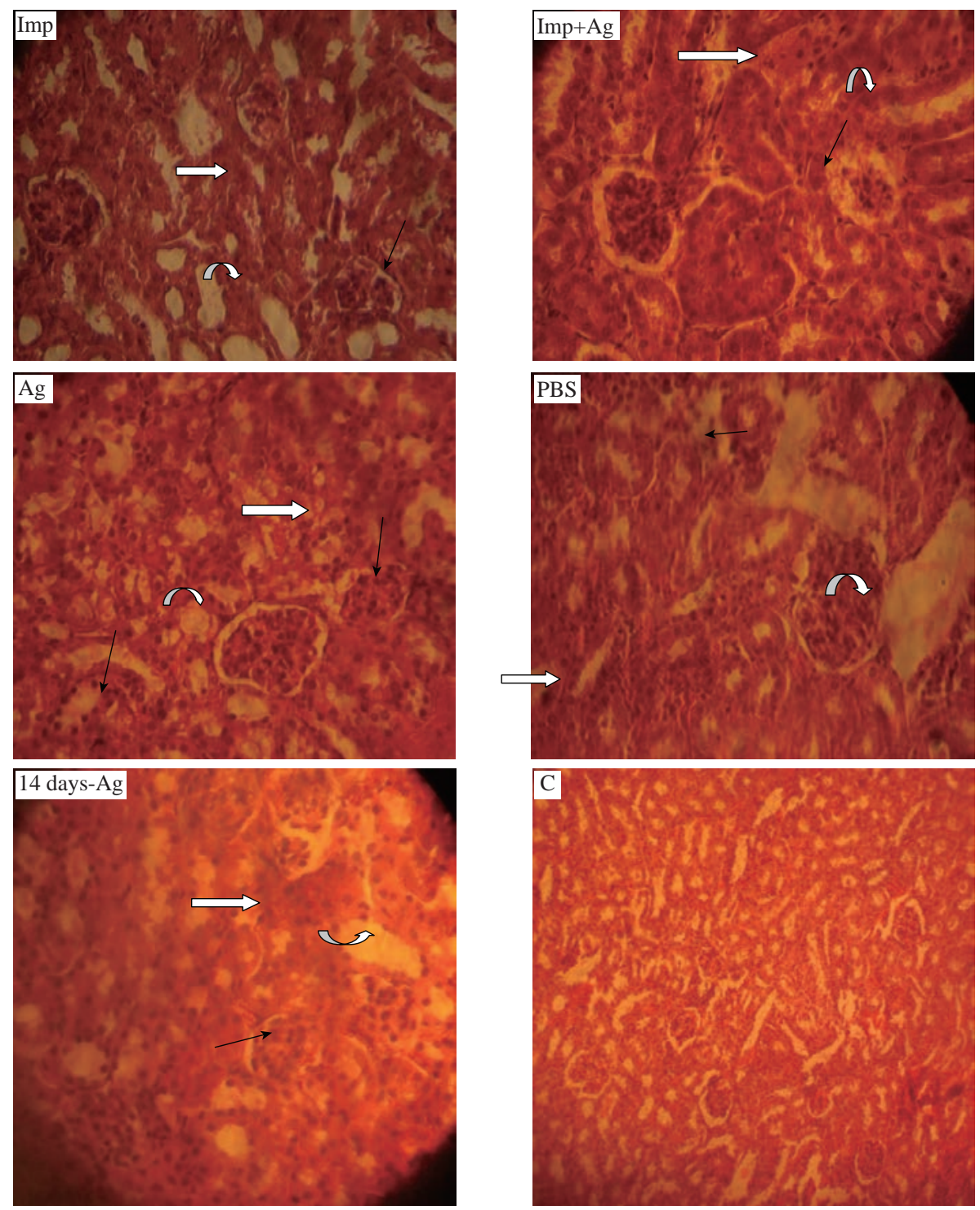

Fig. 7 Histological sections of mice kidney $(40 \times)$. Imp: Imipenem $(10 \mathrm{mg} / \mathrm{kg})$; Imp+Ag: Imipenem plus Cit-AgNPs combination $(5$ mg + 1.75 mg /kg); Ag: Cit-AgNPs (3.5 mg/kg); PBS: Phosphate buffer saline receiving mice for 3 days; 14 days-Ag: Cit-AgNPs (3.5 $\mathrm{mg} / \mathrm{kg}$ ) receiving mice for 3 days and sacrificed after 14 days. C: normal healthy control. Atrophy and loss of glomeruli structure (black arrows), necrosis and degeneration proximal and distal convoluted tubules (white arrows), and mucin secretion (curved arrows) are seen in all sections except healthy control. 
previously [31]. At a relatively high dose, AgNps induce several histopathological changes represented by hemorrhage, severe atrophy of glomeruli, loss of morphology, degeneration, congestion of proximal, distal convoluted tubules, and Henley loop epithelial cell distraction. The severity of these changes depends on the administrated dose of AgNPs [29].

The severe signs and lesions of PBS treated groups are due to $\mathrm{A}$. baumannii infection hence no other possible causes were found. After 14 days of high dose $(3.5 \mathrm{mg} / \mathrm{kg})$ Cit-AgNPs administration, the lesions appeared to be less than lesions of mice groups treated with Cit-AgNPs and PBS but the evidence of degeneration, necrosis, and mucin secretion is still visible (Fig. 7). The excretion of AgNPs from the body is slow and delayed and it was observed that the level of AgNPs in urine is high until day 14 then AgNPs concentration was a decline [30]. The slow AgNPs excretion may explain the delay and slow regeneration of kidney tissue. On the other hand, the excretion routes of AgNPs depend on the size of the nanoparticles. Small size AgNPs are excreted mainly through the urinary tract while larger size through the liver then out with feces [32].

Spleen and liver are the main targets for the accumulation of AgNPs. Phagocytes, like spleen B cells and macrophages and liver Kupffer cells, play a major role in such a process [33]. Regarding the spleens of the groups, the spleen of the imipenem treated group showed well define tissue and normal red
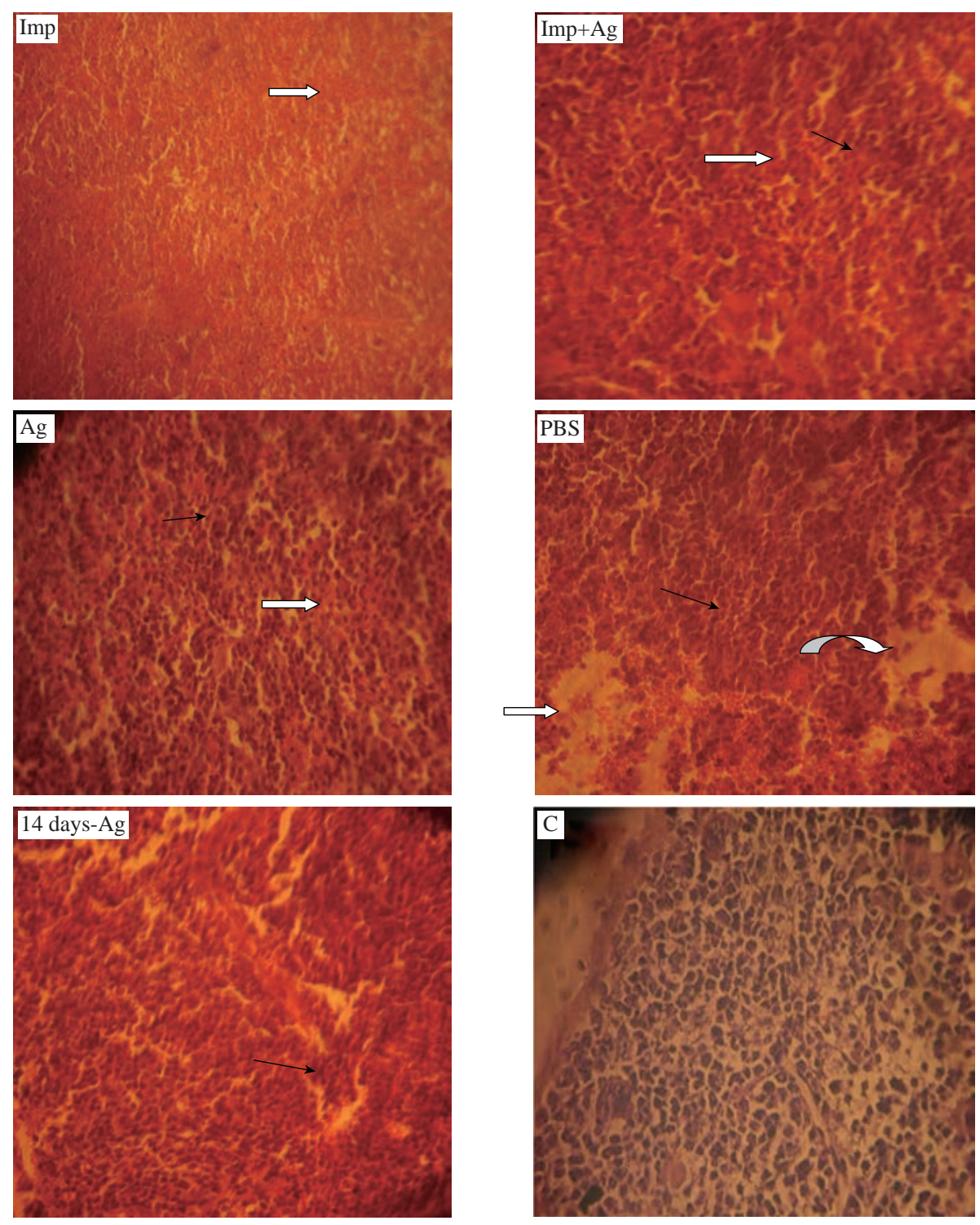

Fig. 8 Histological sections of mice spleen $(40 \times)$. Imp: Imipenem $(10 \mathrm{mg} / \mathrm{kg})$; Imp+Ag: Imipenem plus Cit-AgNPs combination $(5$ mg +1.75 mg/kg); Ag: Cit-AgNPs (3.5 mg/kg); PBS: Phosphate buffer saline receiving mice for 3 days; 14 days-Ag: Cit-AgNPs (3.5 $\mathrm{mg} / \mathrm{kg}$ ) receiving mice for 3 days and sacrificed after 14 days; C: Normal healthy control. Necrosis and degeneration (black arrows), interstitial bleeding (white arrows), and massive tissue distraction (curved arrow) are seen. 
and white pulps and trabeculae but there is bleeding throughout the tissue. Imipenem plus Cit-AgNPs group showed bleeding and loss of red and white pulps structures. The same was observed in the spleen of the group treated with Cit-AgNPs. Cit-AgNPs have a toxic effect on the spleen at both low and high doses hence the severity of the increased lesion as compared with imipenem treated. The $20 \mathrm{~nm}$ AgNPs were mainly deposited into the liver, the kidneys, and spleen, whereas the larger AgNPs were mainly distributed to the spleen followed by the liver and lung after injection of Cit-AgNPs [31]. Most Cit-AgNPs of this study are relatively large particles (50\% of Cit-AgNPs size was $60 \mathrm{~nm}$ ) which may explain spleen toxicity. Phosphate buffer-treated mice showed massive tissue distraction,
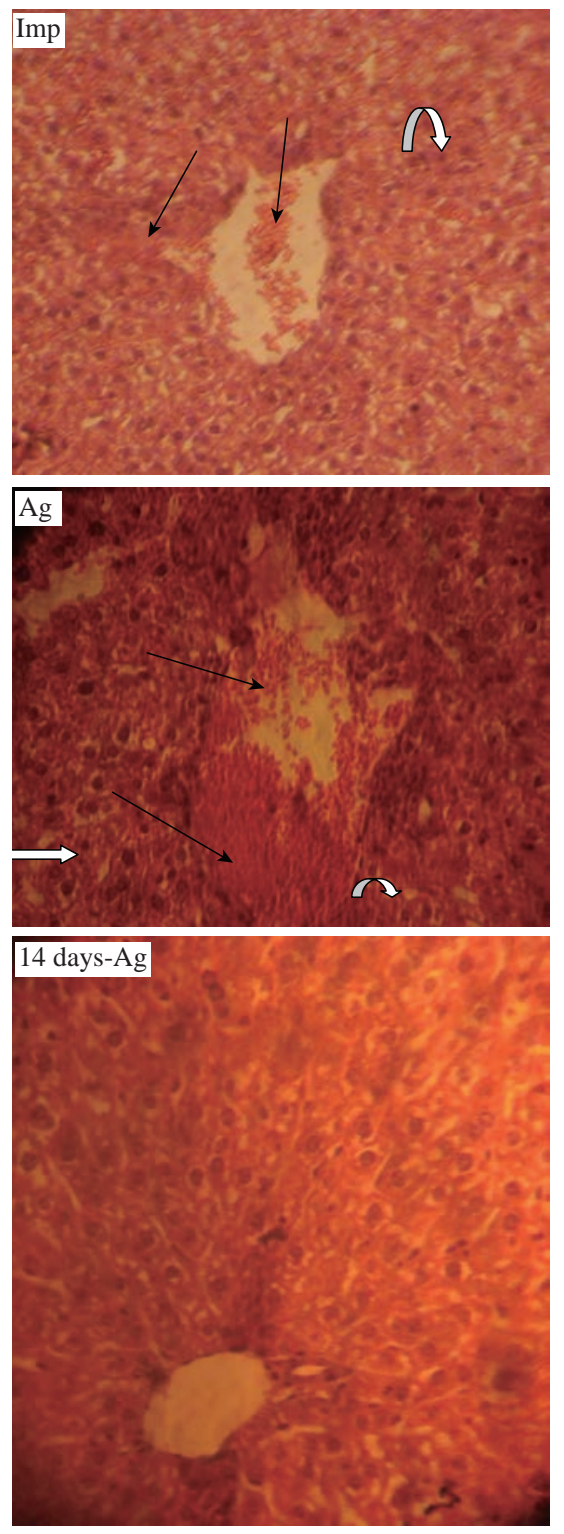

severe bleeding, and necrosis. In the group treated with Cit-AgNPs for 14 days, no bleeding was observed and evidence of regeneration is begun (Fig. 8).

The livers of the group treated with imipenem were severely congested. Degeneration, necrosis, and bleeding are also observed this may be contributed to A. baumannii infection. While livers of groups treated imipenem plus Cit-AgNPs, AgNPs only, and PBS showed severe congestion, loss of tissue structure, necrosis as well as inclusion body degeneration and swelling degeneration and bleeding. The effect of both A. baumannii infection and Cit-AgNPs maybe double the toxic effect. The severity of these lesions is obvious in AgNPs and PBS that showed severe bleeding and necrosis. After 14 days of AgNPs treatment, the liver
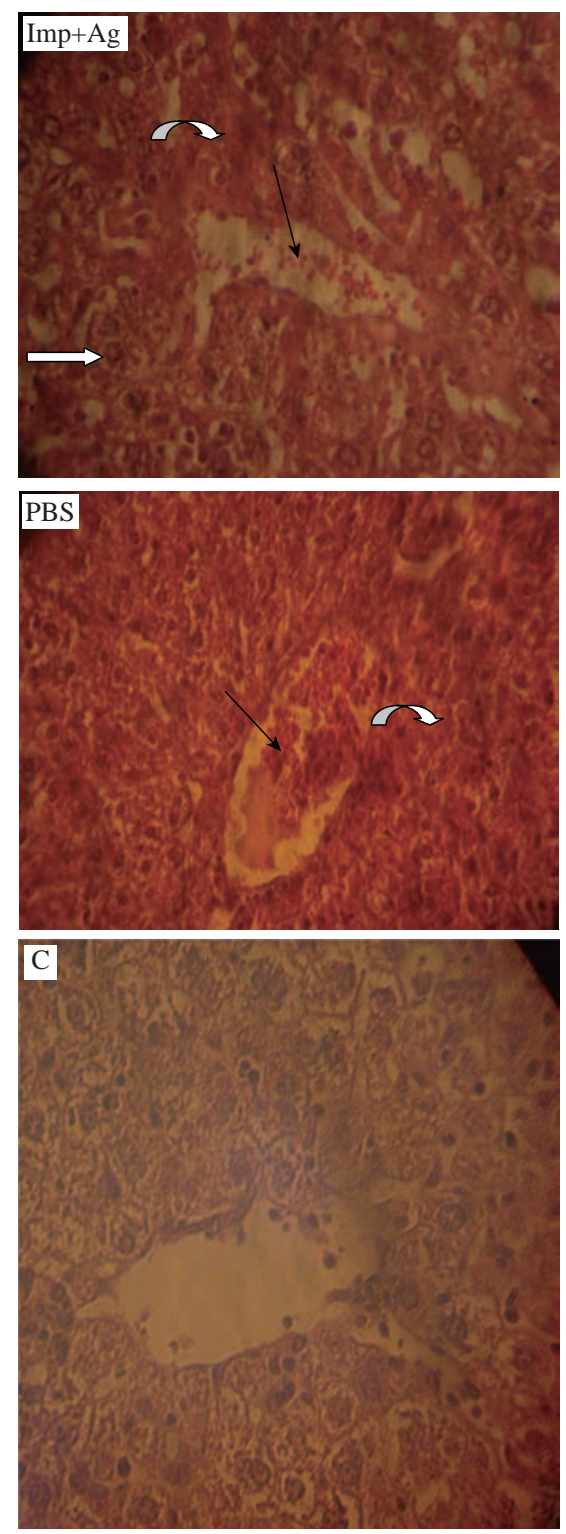

Fig. 9 Histological sections of mice liver $(40 \times)$. Imp: Imipenem $(10 \mathrm{mg} / \mathrm{kg})$; Imp+Ag: Imipenem plus Cit-AgNPs combination $(5$ $\mathrm{mg}+1.75 \mathrm{mg} / \mathrm{kg}$ ); Ag: Cit-AgNPs (3.5 mg/kg); PBS: Phosphate buffer saline receiving mice for 3 days; 14 days-Ag: Cit-AgNPs (3.5 $\mathrm{mg} / \mathrm{kg}$ ) receiving mice for 3 days and sacrificed after 14 days; C: Normal healthy control. Severe congestion and bleeding especially in Ag treated mice liver (black arrows), necrosis (curved arrows), and inclusion body degeneration (white arrows) are seen. 
was normal as the control mice group, and no evidence of tissue inflammation was observed (Fig. 9). This finding may be due to the amazing ability of the liver to generate and heal [34]. Moreover, AgNPs induce the expression of some genes related to apoptosis and oxidative stress [35, 36].

In conclusion, Cit-capped AgNPs have antibacterial activity against MDR A. baumannii but not against K. pneumoniae and E. coli. Cit-capped AgNPs increase the inhibition zone of imipenem in additive effect and MIC and MBC of Cit-capped AgNPs are relatively low. Cit-capped AgNPs eliminate A. baumannii infection in vivo when it was given alone or in combination with imipenem. The cytotoxicity of Cit-AgNPs is dosedependent and the organs differ in the illumination of the inflammatory effect of Cit-AgNPs after high dose administration.

\section{Acknowledgments}

The authors are thankful to Mustansiriyah University (www.uomustansiriyah.edu.iq) for supporting. Mustansiriyah university is the funding source for the current article.

\section{Conflict of Interests}

The authors declare that no competing interest exists.

\section{References}

[1] O.A. Ojo, I.I. Olayide, M.C. Akalabu, et al., Nanoparticles and their Biomedical Applications. Biointerface Research in Applied Chemistry. 2020, 11(1): 8431-8445.

[2] H.F.M. Xavier, V.M. Nadar, P. Patel, et al., Selective antibacterial and apoptosis-inducing effects of hybrid gold nanoparticles - A green approach. Journal of Drug Delivery Science and Technology. 2020, 59: 101890.

[3] D.P. Gnanadhas, M.B.Thomas, R. Thomas, et al., Interaction of Silver Nanoparticles with Serum Proteins Affects Their Antimicrobial Activity In Vivo. Antimicrobial Agents and Chemotherapy. 2013, 57 (10): 4945-4955.

[4] J.R. Morones, J.L. Elechiguerra, A. Camacho, et al., The bactericidal effect of silver nanoparticles. Nanotechnololgy. 2005, 16: 2346-2353.

[5] C.N. Lok, C.M. Ho, R. Chen, et al., Silver nanoparticles: partial oxidation and antibacterial activities. Journal of Biological Inorganic Chemistry. 2007, 12: 527-534.

[6] M. Rai, A. Yadav, and A. Gade, Silver nanoparticles as a new generation of antimicrobials. Biotechnolology Advances. 2009, 27: 76-83.

[7] J.M. Lee, D. Kim, and T.H. Kim, Facile route for preparation of silica-silver heterogeneous nanocomposite particles using alcohol reduction method. Matererials
Letters. 2007, 61: 1558-1562.

[8] D.V. Quang, P.B. Sarawade, A. Hilonga, et al., Facile route for preparation of silver nanoparticle-coated precipitated silica. Applied Surface Science. 2011, 257: 4250-4256.

[9] N.H. Zaki, Z. Husain, Enhanced antibacterial and antibiofilm activities of biosynthesized silver nanoparticles against pathogenic bacteria. Journal of Genetic and Environmental Resources. 2016, 4(2): 197-203.

[10] E. Amato, Y.A. Diaz-Fernandez, A. Taglietti, et al., Synthesis, characterization and antibacterial activity against Gram-positive and Gram-negative bacteria of biomimetically coated silver nanoparticles. Langmuir. 2011, 27: 9165-9173.

[11] A.M. AbdelMohsen, R. Hrdina, L. Burgert, et al., Antibacterial activity and cell viability of hyaluronan fiber with silver nanoparticles. Carbohydrate Polymers. 2013, 92: 1177-1187.

[12] I. Sondi, B. Salopek-Sondi, Silver nanoparticles as antimicrobial agent: a case study on E. coli as a model for Gram-negative bacteria. Journal of Colloid and Interface Science. 2004, 275: 177-182.

[13] Q.L. Feng, J. Wu, G.Q. Chen, et al., A mechanistic study of the antibacterial effect of silver ions on Escherichia coli and Staphylococcus aureus. Journal of Biomedical Materials Research. 2000, 52: 662-668.

[14] Y. Matsumura, K. Yoshikata, S.I. Kunisaki, et al., Mode of bactericidal action of silver zeolite and its comparison with that of silver nitrate. Applied and Environmental Microbiology. 2003, 69: 4278-4281.

[15] P. Dibrov, J. Dzioba, K.K. Gosink, et al., Chemiosmotic mechanism of antimicrobial activity of $\mathrm{Ag}+$ in Vibrio cholerae. Antimicrobial Agents and Chemotherapy. 2002, 46: $2668-2670$.

[16] C.N. Lok, C.M. Ho, R. Chen, et al., Proteomic analysis of the mode of antibacterial action of silver nanoparticles. Journal of Proteome Research. 2006, 5: 916-924.

[17] Z.S. Pillai, P.V. Kamat, What factors control the size and shape of silver nanoparticles in the citrate ion reduction method? Journal of Physical Chemistry. 2004, 108: 945951.

[18] S.G.H. Bartual, C. Seifert, M.A.D. Hippler, et al., Development of a Multilocus Sequence Typing Scheme for Characterization of Clinical Isolates of Acinetobacter baumannii. Journal of Clinical Microbiology. 2005, 43(9): 4382-4390.

[19] I.G. Auda, S.N. Al-Kakei, and S.L. Hamed, Occurrence of CTX-M-I Type $\beta$-lactamases Gene in Certain GramNegative Bacteria. Iraqi Postgraduate Medical Journal. 2013, 12(2): 306-311.

[20] CLSI., Performance standards for antimicrobial susceptibility testing; Twenty-Third informational supplement. M100-S23.33: 1. 2013.

[21] A.M. Atta, A.A. Al-Lohedan, and A.O. Ezzat, Synthesis of silver nanoparticles by green method stabilized to synthetic human stomach fluid. Molecules, 2014, 19: 6737-6753.

[22] Y. Sugimoto, P. Pou, and M. Abe, Chemical identification of individual surface atoms by atomic force microscopy. Nature, 2007, 446(7131): 64-67.

[23] I. Wiegand, K. Hilpert, and R.E. Hancock, Agar and broth dilution methods to determine the minimal inhibitory concentration (MIC) of antimicrobial substances. Nature protocols, 2008, 3(2): 163.

[24] M. Lin, C. Lan, Antimicrobial resistance in Acinetobacter baumannii: From bench to bedside. World Journal of Clinical Cases, 2014, 2(12): 787-814.

[25] G. Zhou, W. Wang, Synthesis of silver nanoparticles and their antiproliferation against human lung cancer cells in vitro. Orient Journal of Chemiatry, 2012, 28(2): 651-655. 
[26] A. Panácek, L. Kvítek, R. Prucek, et al., Silver colloid nanoparticles: synthesis, characterization, and their antibacterial activity. Journal of Physical Chemistry. 2006, 110: $16248-16253$.

[27] K. Loeschner, N. Hadrup, K. Qvortrup, et al., Distribution of silver in rats following 28 days of repeated oral exposure to silver nanoparticles or silver acetate. Particle and Fibre Toxicology. 2011, 8: 18.

[28] K. Park, E.J. Park, I. Chun, et al., Bioavailability and toxicokinetics of citrate-coated silver nanoparticles in rats. Archives of Pharmacal Research, 2011, 34(1): 153158.

[29] P.J. Prakash, S. Royana, andf P. Sankarsan, Small size silver nanoparticle's corrosive and hazardous manifestations on mature and developing kidney following accumulation in pregnant mice and offspring's after serial oral bolus experimental application: A new chapter in teratogenicity and toxicity search. Journal of Cytology and Histology, 2017, 8: 467.

[30] K. Dziendzikowska, J. Gromadzka-Ostowska, and A. Lankoff, Time-dependent biodistribution and excretion of silver nanoparticles in male Wistar rats. Journal of Applied Toxicology, 2012, 32: 920-928.

[31] Y. Lee, P. Kim, J. Yoon, et al., Serum kinetics, distribution and excretion of silver in rabbits following 28 days after a single intravenous injection of silver nanoparticles. Nanotoxicology, 2013, 7(6): 1120-1130.
[32] H.S. Choi, W. Liu, P. Misra, et al., Renal clearance of quantum dots. Nature Biotechnology, 2007, 25: 11651170.

[33] Y. Xue, S. Zhang, Y. Huang, et al., Acute toxic effects and gender-related biokinetics of silver nanoparticles following an intravenous injection in mice. Journal of Applied Toxicology, 2012, 32(11): 890-899.

[34] M. Harihara, Tissue repair: An important determinant of final outcome of toxicant-induced injury. Toxicologic Pathology, 2005, 33(1): 41-51.

[35] J. Hou, L. Zhao, H. Tang, et al., Silver nanoparticles induced oxidative stress and mitochondrial injuries mediated autophagy in HC11 cells through Akt/AMPK/ mTOR pathway. Bioloical Trace Elemement Research. 2021, 199: 1062-1073.

[36] D. Kovács, N. Igaz, C. Keskeny, et al., Silver nanoparticles defeat p53-positive and p53-negative osteosarcoma cells by triggering mitochondrial stress and apoptosis. Scientific Reports, 2016, 6: 27902.

Copyright $@$ Ibtesam Ghadban Auda, Istabreq Mohamed Ali Salman, Dalal Abed Al-Sattar, and Jameelah Ghadban Oduha. This is an open-access article distributed under the terms of the Creative Commons Attribution License, which permits unrestricted use, distribution, and reproduction in any medium, provided the original author and source are credited. 